\title{
AN UPDATE ON THE MANAGEMENT OF URINARY TRACT INFECTIONS IN THE ERA OF ANTIMICROBIAL RESISTANCE ${ }^{1}$
}

\author{
Dina Suhail M .Saleh \\ High Diplom Pharmacology \\ University Al Yarmuk, College of Pharmacy
}

DOI: $10.37648 /$ ijrst.v10i02.002

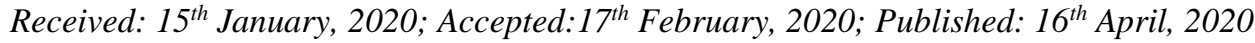

\begin{abstract}
Urinary tract infections (UTIs) caused by antibiotic-resistant Gram-negative bacteria are a growing concern due to limited therapeutic options. Gram-negative bacteria, specifically Enterobacteriaceae, are common causes of both community-acquired and hospital acquired UTIs. These organisms can acquire genes that encode for multiple antibiotic resistance mechanisms, including extended-spectrum-lactamases (ESBLs), AmpC- $\beta$-lactamase, and carbapenems. The assessment of suspected UTI includes identification of characteristic symptoms or signs, urinalysis, dipstick or microscopic tests, and urine culture if indicated. UTIs are categorized according to location (upper versus lower urinary tract) and severity (uncomplicated versus complicated). Increasing rates of antibiotic resistance necessitate judicious use of antibiotics through the application of antimicrobial stewardship principles. Knowledge of the common causative pathogens of UTIs including local susceptibility patterns are essential in determining appropriate empiric therapy. The recommended first-line empiric therapies for acute uncomplicated bacterial cystitis in otherwise healthy adult nonpregnant females is a 5-day course of nitrofurantoin or a 3-g single dose of Fosfomycin tromethamine. Second-line options include fluoroquinolones and $\beta$-lactams, such as amoxicillinclavulanate. Current treatment options for UTIs due to AmpC- $\beta$-lactamase-producing organisms include Fosfomycin, nitrofurantoin, fluoroquinolones, cefepime, piperacillin-tazobactam and carbapenems. In addition, treatment options for UTIs due to ESBLs-producing Enterobacteriaceae include nitrofurantoin, Fosfomycin, fluoroquinolones, cefoxitin, piperacillin-tazobactam, carbapenems, ceftazidime-avibactam, ceftolozane-tazobactam, and aminoglycosides. Based on identification and susceptibility results, alternatives to carbapenems may be used to treat mild-moderate UTIs caused by ESBL-producing Enterobacteriaceae. Ceftazidime-avibactam, colistin, polymyxin B, Fosfomycin, aztreonam, aminoglycosides, and tigecycline are treatment options for UTIs caused by carbapenem-resistant Enterobacteriaceae (CRE). Treatment options for UTIs caused by multidrug resistant (MDR)Pseudomonas spp. include fluoroquinolones, ceftazidime, cefepime, piperacillin-tazobactam, carbapenems, aminoglycosides, colistin, ceftazidime-avibactam, and cefdaloxime-tazobactam. The use of fluoroquinolones for empiric treatment of UTIs should be restricted due to increased rates of resistance. Aminoglycosides, colistin, and tigecycline are considered alternatives in the setting of MDR Gram-negative infections in patients with limited therapeutic options.
\end{abstract}

${ }^{1}$ How to cite the article: Saleh D.S.M., An Update on the Management of Urinary Tract Infections in the Era of Microbial Resistance, IJRST, Apr-Jun 2020, Vol 10, Issue 2, 8-15, DOI: http://doi.org/10.37648/ijrst.v10i02.002 
Keywords: Antibiotic resistance; Enterobacteriaceae; Gram-negative bacteria; cystitis; pyelonephritis; urinary tract infections

\section{INTRODUCTION}

A urinary tract infection (UTI) is an impurity that imitates a part of the urinary tract.(1) it is mostly affects main part of lower urinary tract. Sometimes, this infection known as a bladder infection (cystitis). in case where is the infection effects on the upper part of urinary tract, it would be known as a kidney infection (pyelonephritis).(2) Several Symptoms may company the a lower urinary tract infection involve frequent urination, pain with urination, burning, and sensation of needs to urinate despite of empty bladder as well as some pain above the pubic bone or in the lower back may be present. while the main symptoms of an upper part infection (kidney infection) include flank pain and fever besides to the symptoms of a lower UTI. Infrequently, patient with UTI may sever from urine appeared as bloody. Among older and the young patients the symptoms may be indefinable or non-specific.(3)

It has been identified that the most usual cause of infection is Escherichia coli. Although other bacteria or fungi can rarely be causing the infection. Risk factors that increase the incidence of infection would be include female anatomy, diabetes, obesity, sexual intercourse and family history.(4) even though, sexual contact would be consider as a risk factor, UTIs are not classified as sexually transmitted infections (STIs). Upper part infection (Kidney infection) it is not common ,but if it occurs, usually following a bladder impurity and it may also occur from a blood borne infection.(5) Determine the infection in healthy women may be based on symptoms only. Diagnostic vague symptoms, diagnosis can be difficult because bacteria may be present without there being an infection. ${ }^{[13]} \mathrm{In}$ complicated cases or if treatment fails, a urine culture may be useful.(6)

A short course of antibiotics treatment could be good path for in uncomplicated cases of UTI as nitrofurantoin or trimethoprim and sulfamethoxazole.(7) Resistance to many of the antibiotics used to treat this condition actually increased. In complicated cases, it may be needed to long course or intravenous antibiotics may be needed.(7) in case, the patient taken regular treatment but symptoms do not improve in two or three days, further diagnostic testing should be recommended. Phenazopyridine may help with symptoms. ${ }^{[1]}$ In those who have bacteria or white blood cells in their urine but have no symptoms, antibiotics are generally not needed, ${ }^{[14]}$ although during pregnancy is an exception. ${ }^{[15]}$ In those with frequent infections, a short course of antibiotics may be taken as soon as symptoms begin or long-term antibiotics may be used as a preventative measure.(6)

Recently it has been reported that around 150 million people develop a urinary tract infection in a given year.(4) this statists approved that UTI specially bacterial infections are more common in women than men. (6) Up to $10 \%$ of women have a urinary tract infection in a given year, and half of women have at least one infection at some point in their lifetime.(8) The prevalence of UTI among women are mostly occur between the ages of 16 and 35 years. (7)

Apathogenic E. coli that are prevalence in the gut is the main source of $80-85 \%$ of community-acquired urinary tract infections. (9) on the other hand, Staphylococcus saprophyticus can be causing around 5-10\%.(9)

UTI that happen due to Staphylococcus aureus normally happen as secondary to blood-borne infections. However it has been diagnostic that Chlamydia trachomatous and Mycoplasma genitalium could infect only urethra but not the bladder. These kind of infections are classified as a urethritis rather than urinary tract infection.(2) The bacteria that cause urinary tract infections typically enter the bladder via the urethra. However, infection may also occur via the blood or lymph. It is believed that the bacteria are usually transmitted to the urethra from the bowel, with females at greater risk due to their anatomy. ${ }^{[6]}$ After gaining entry to the 
bladder, E. Coli are able to attach to the bladder wall and form a biofilm that resists the body's immune response. (9)

Most of straightforward cases, a diagnosis can make and treatment given upon on symptoms alone without further laboratory confirmation while in complicated or questionable cases, it is very useful to confirm the diagnosis through sending patients to do urinalysis, looking for the presence of urinary nitrites, white blood cells (leukocytes), or leukocyte esterase or even send them to urine microscopy, if needed. Urine culture is supposed to be positive if it shows a bacterial colony count of greater than or equal to $10^{3}$ colony-forming units per $\mathrm{mL}$ of a typical urinary tract organism. (10)

In complicated cases antibiotic sensitivity also can be tested in culture. the outcome from culturing are useful in the selection of antibiotic treatment. However, women with negative cultures may still improve with antibiotic treatment.(10)

Uncomplicated infections can be diagnosed and treated based on symptom alone. Antibiotics taken by mouth

such

as trimethoprim/sulfamethoxazole (TMP/SMX), nitro furantoin, or Fosfomycin are typically first line. Moreover, Cephalosporins, amoxicillin/clavulanic acid, or a fluoroquinolone can also be utilized. However, resistance to fluoroquinolones among some of bacteria that produce urinary infections has been growing. Increase risk of serious side effects of fluoroquinolones ,the Food and Drug Administration (FDA) recommends against the use of fluoroquinolones when other options are available. (11) These medications substantially shorten the time to recovery with all being equally effective. A threeday treatment with trimethoprim, TMP/SMX, or a fluoroquinolone is usually sufficient, whereas nitrofurantoin requires 5-7 days. Fosfomycin may be used as a single dose but has been associated with lower rates of efficacy.(11)

With treatment, symptoms should improve within 36 hours. 50\% of patients would be recovered without using any drug or specific treatments within a few days or weeks. Fluoroquinolones are not recommended as a first treatment. The Infectious Diseases Society of America states this due to the concern of generating resistance to this class of medication. Amoxicillin -clavulanate plays less effectual than other options. Despite this precaution, some resistance has developed to all of these medications related to their widespread use.(12) Trimethoprim alone is deemed to be equivalent to TMP/SMX in some countries. For simple UTIs, children often respond to a three-day course of antibiotics. Women with recurrent simple UTIs are over $90 \%$ accurate in identifying new infections. They may benefit from self-treatment upon occurrence of symptoms with medical follow-up only if the initial treatment fails.(13)

Complicated UTIs are more problematic to treat and frequently wants more aggressive assessment, treatment and follow-up. It might need identifying and focusing the underlying complication. in now days, aggregate antibiotic resistance is producing concern about the future of treating those with complicated and recurrent UTI.(14). In current study authors investigate gender and age of patients suffering from UTI and which bacteria is more prevalence with study the resistance of several common and new antibiotic.

\section{MATERIAL AND METHODS}

study was conducted in Al-Numman teaching Hospital, Baghdad Iraq. the study conducted after getting approval from the institution ethical.

Number of patients was 50 of they were recruited from November till March 2019. patients ages were between 1-70 years old. Analysis was done using the statistical analysis system SAS 2012. Program was effect of difference factors in study parameters chiSquare test to find out the significant compare between percentages in the current study.

many information and data collected about patient's demographic such as age, gender, a.u (epithalami cell).

urine culture done for each participant to identified microorganism, antibiotics, resistance of antibiotics. 


\section{RESULTS AND DISCUSSION:}

The incidence of UTI and its clinical impact varies for both sexes and for different stages of life in present study it has been shown in table $1 \& 2$. In table 1, higher prevalence of UTIs was in females $62 \%$. Compared to male $38 \%$. These results are agreement with other reports made in Baghdad teaching hospital- Iraq in 2016 which showed that UTIs are very common in women in percentage $65 \%$ than in male $34 \%$.(15)

Sexual activity is reason of about $75-90 \%$ of bladder infections among young sexually active women and this sort of infection mostly to related with the risk of infection related to frequency of sex times. The medical term which is known among doctors as "honeymoon cystitis" identified to UTI phenomenon through of early stages of marriage. However, In post-menopausal stage of female life , sexual activity does not affect the risk of developing a UTI. in addition to frequent of sex, there are several risk factors that increase the infection including Spermicide use, independent of sexual frequency, Diaphragm , Condom use without spermicide or use of birth control pills. (13)

Furthermore, female are more prone to infections because urethra in females is considerably short and close to the anus comparing to male. in addition, estrogen hormone levels in women are dropped with menopause, her risk of urinary tract infections increases due to the loss of protective vaginal flora.(16)

Moreover, we can notice from table 2 that number of incidences would be increased with patients at more than 40 years old where the number was higher among them to reach 14 cases at $28 \%$. As well as same table showed that younger patient who age 1-10 have high record of UTI which reached 11 cases at $22 \%$ among sample of current study.

Figure 1 showed that E-coli was the most prevalent organism cause UTI among patients included in the current study and this conclusion found in other research done in Baghdad hospital 2015 E-coli as a commonest cause of UTI. (17) UTI infections may be done to certain virulence factors like hemolysin production and presence of fimbriae the most organism caused UTI. (18) In this study were belong gram negative bacteria which were isolated from74. Patients these results were almost similar to those of Baghdad teaching hospital samples that are collected in previous studies $(19,20)$

In addition, our findings demonstrate that bacterial involvement in UTI is E-coli followed by staph aureus then entry bacteria and pseudomonas with a smaller number of cases there was klebsiellaspp as show in figure 1. Pervious study showed same and differ sort of bacteria with different percentage of causes where healthcareassociated urinary tract infections (mostly related to urinary catheterization) involve a much broader range of pathogens including: $E$. coli (27\%), Klebsiella (11\%), Pseudomonas (11\%), the fungal pathogen Candida albicans $(9 \%)$, and Enterococcus (7\%). (21)

The results from current study, especially with resistance test, displaced that amikacin is more effective against E-coli followed by gentamycin then floxacillin then piperacillin tazobactam and the last in study is cefixime figure 2

Other studies found that amikacin also effective against staph aureus. cefixime was fewer sensitive antibiotics to the E-coli due to misuse of this antibiotics and patient not complete antibiotics dosage regimen $(22,23)$

Moreover, figure 2 illustrated that ceftriaxone is more resistant to E-coli with comparable results to other study data from AL- Kadhimiah teaching hospital where we found 12 case eight of them resistance to ceftriaxone due to utilize of inactivate antimicrobial lacking of quality control on some sources of antibiotics entering Iraq especially by the private sector. $(24,25)$

it has been suggested that $\mathrm{E}$ - coli is the most common microorganism, followed by Klebsiella and Proteus spp. leading to UTI. Klebsiella and Proteus spp., are also frequently associated with stone disease. The presence of Gram 
positive bacteria such as Enterococcus and Staphylococcus are increased recently as source of UTI.(26)

conclude high rate of resistances occurred in local pathogens. These results is controversy to the studies reported previously.

The use of the antibiotics needs to be under heat assessment and control with lab investigation to

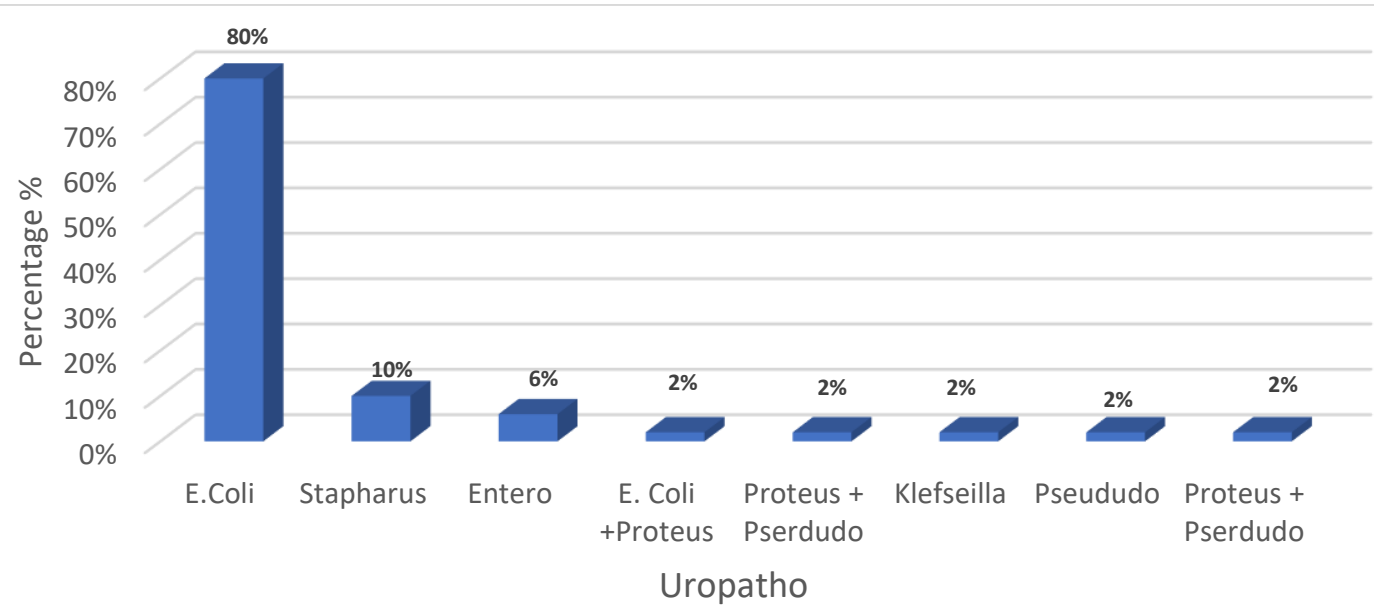

Figure 1: Distribution of Sample study according to Uropathy

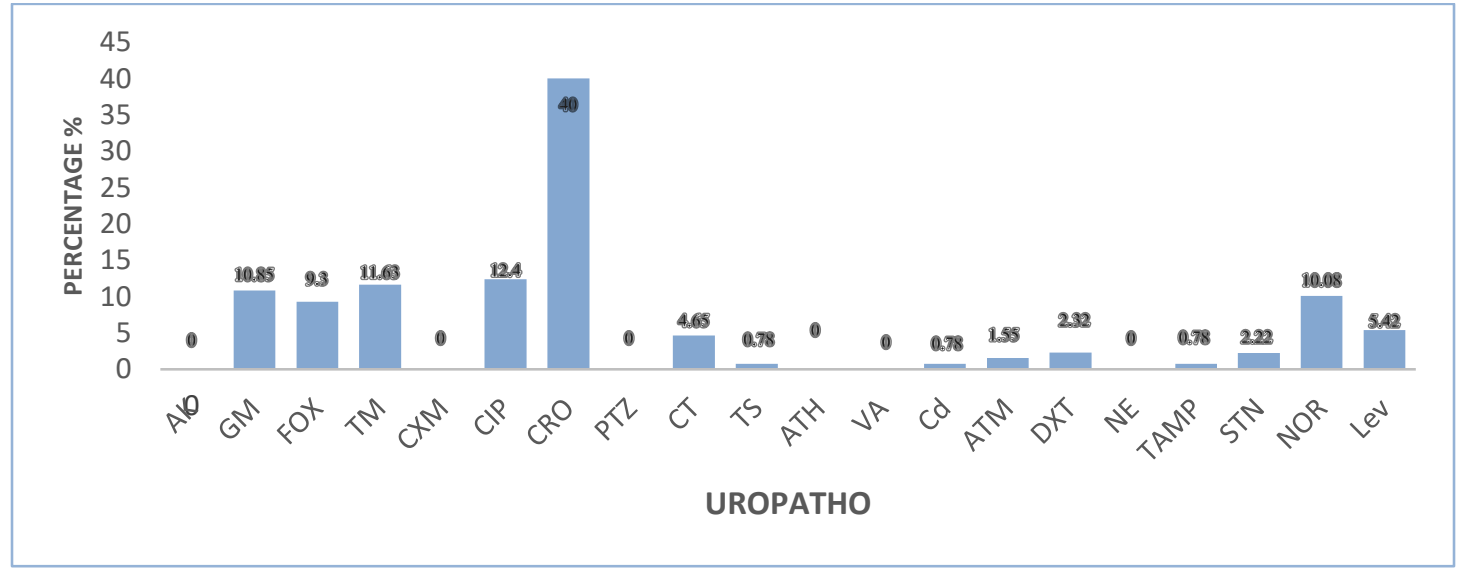

Figure 2: Distribution of sample study according to the resistance 
Table 1: Distribution of sample study according to gender.

\begin{tabular}{|l|l|l|}
\hline Gender & No & Percentage \% \\
\hline Male & 19 & 38.00 \\
\hline Female & 31 & 62.00 \\
\hline Total & 50 & $100 \%$ \\
\hline Chi-Square(X) & ----- & $9427^{* *}$ \\
\hline P-value & ----- & 0.0002 \\
\hline *** $(\mathbf{P}<\mathbf{0 . 0 1})$ & & \\
\hline
\end{tabular}

Table 2: Distribution of Sample study according to Age of Groups

\begin{tabular}{|l|l|l|}
\hline Age of groups & No & 10.00 \\
\hline Less than 1 year & 5 & 22.00 \\
\hline 1-10 years & 11 & 12.00 \\
\hline 11-2- years & 6 & 16.00 \\
\hline 21-30 years & 8 & 12.00 \\
\hline 31-40 years & 6 & 28.00 \\
\hline More than 40 & 14 & $100 \%$ \\
\hline Total & 50 & $7.944^{* *}$ \\
\hline Chi-Square & ---- & 0.00874 \\
\hline P- value & ---- & \\
\hline ** (P<0.01) & & \\
\hline
\end{tabular}

Conflict of interest: There is none.

Funding: self-funded

Data Availability: Is available on request 
Ethics Statement: the authors declare that there are not conflict of interest for all the authors

\section{REFERENCES}

1. Peach BC, Garvan GJ, Garvan CS, Cimiotti JP. Risk Factors for Urosepsis in Older Adults: A Systematic Review. Gerontol Geriatr Med. 2016;2:2333721416638980-.

2. Lane DR, Takhar SS. Diagnosis and management of urinary tract infection and pyelonephritis. Emerg Med Clin North Am. 2011;29(3):539-52.

3. Franco AV. Recurrent urinary tract infections. Best Pract Res Clin Obstet Gynaecol. 2005;19(6):861-73.

4. Flores-Mireles AL, Walker JN, Caparon M, Hultgren SJ. Urinary tract infections: epidemiology, mechanisms of infection and treatment options. Nat Rev Microbiol. 2015;13(5):269-84.

5. Linton AD. Introduction to medical-surgical nursing: Elsevier Health Sciences; 2015.

6. Colgan R, Williams M, Johnson JR. Diagnosis and treatment of acute pyelonephritis in women. American family physician. 2011;84(5):519.

7. Salvatore S, Salvatore S, Cattoni E, Siesto G, Serati M, Sorice P, et al. Urinary tract infections in women. European journal of obstetrics \& gynecology and reproductive biology. 2011;156(2):131-6.

8. Zhanel GG, Golden AR, Zelenitsky S, Wiebe K, Lawrence CK, Adam HJ, et al. Cefiderocol: A Siderophore Cephalosporin with Activity Against Carbapenem-Resistant and Multidrug-Resistant Gram-Negative Bacilli. Drugs. 2019;79(3):271-89.

9. Nace DA, Perera SK, Hanlon JT, Saracco S, Anderson G, Schweon SJ, et al. The Improving Outcomes of UTI Management in Long-Term Care Project (IOU) Consensus Guidelines for the Diagnosis of Uncomplicated Cystitis in Nursing Home Residents. J Am Med Dir Assoc. 2018;19(9):765-9.e3.

10. Woodford HJ, George J. Diagnosis and management of urinary infections in older people. Clinical medicine. 2011;11(1):80-3.

11. Trestioreanu AZ, Green H, Paul M, Yaphe J, Leibovici L. Antimicrobial agents for treating uncomplicated urinary tract infection in women. Cochrane database of systematic reviews. 2010(10).

12. Jarvis TR, Chan L, Gottlieb T. Assessment and management of lower urinary tract infection in adults. Aust Prescr. 2014;37:7-9.

13. Nicolle LE. Uncomplicated urinary tract infection in adults including uncomplicated pyelonephritis. Urol Clin North Am. 2008;35(1):1-12, v.

14. Hassan MHA. Effect of intervention guidelines on self care practices of pregnant women with urinary tract infection. Life Science Journal. 2015;12(1):113-24.

15. Kassim K, Khalaf Z, Abdulhassan A, Salman N. Prevalence and Antibiotic Resistance of Bacteria Isolated from Urinary Tract Infections of Pregnant Women in Baghdad Hospitals. Biomedical and Pharmacology Journal. 2018;11:1989-94.

16. Schaechter M, Medoff G, Eisenstein BI. Mechanisms of microbial disease: Lippincott Williams \& Wilkins; 1993.

17. Salmani H, Azarnezhad A, Fayazi MR, Hosseini A. Pathotypic and Phylogenetic Study of Diarrheagenic Escherichia coli and Uropathogenic E. coli Using Multiplex Polymerase Chain Reaction. Jundishapur J Microbiol. 2016;9(2):e28331.

18. Shah C, Baral R, Bartaula B, Shrestha LB. Virulence factors of uropathogenic Escherichia coli (UPEC) and correlation with antimicrobial resistance. BMC Microbiology. 2019;19(1):204.

19. Al-Naqshbandi AA, Chawsheen MA, Abdulqader HH. Prevalence and antimicrobial susceptibility of bacterial pathogens isolated from urine specimens received in rizgary hospital - Erbil. J Infect Public Health. 2019;12(3):330-6.

20. Hassan KI, Abdullah SR. Detection of Pseudomonas aeruginosa in Clinical Samples Using PCR Targeting ETA and gyrB Genes. Baghdad Science Journal. 2018;15(4):401-5. 
21. Sievert DM, Ricks P, Edwards JR, Schneider A, Patel J, Srinivasan A, et al. Antimicrobial-resistant pathogens associated with healthcare-associated infections: summary of data reported to the National Healthcare Safety Network at the Centers for Disease Control and Prevention, 2009-2010. Infect Control Hosp Epidemiol. 2013;34(1):1-14.

22. Fair RJ, Tor Y. Antibiotics and bacterial resistance in the 21st century. Perspect Medicin Chem. 2014;6:2564.

23. Ramirez MS, Tolmasky ME. Amikacin: Uses, Resistance, and Prospects for Inhibition. Molecules. 2017;22(12):2267.

24. Kraemer SA, Ramachandran A, Perron GG. Antibiotic Pollution in the Environment: From Microbial Ecology to Public Policy. Microorganisms. 2019;7(6):180.

25. Li B, Webster TJ. Bacteria antibiotic resistance: New challenges and opportunities for implant-associated orthopedic infections. J Orthop Res. 2018;36(1):22-32.

26. Opperman E. Cranberry is not effective for the prevention or treatment of urinary tract infections in individuals with spinal cord injury. Spinal cord. 2010;48(6):451. 